after tumor growth, and evaluated mRNA and protein expression of quiescence-related markers including FBXW7 in vivo.

Results: In vitro, GRPs showed high expression of stem cell marker CD133 and quiescence-related markers including FBXW7 and low expression of c-Myc at protein level. Cell cycle analysis revealed that majority of GRPs existed in G0/G1 phase. Silencing of FBXW7 gene reduced CD133-positive cell population in GRPs. Knockdown of FBXW7 also increased susceptibility of cells to gefitinib, reversed population of G0/G1-arrest cells to G2/S/M cells, and decreased cell number of GRPs. In vivo, GRTs after gefitinib treatment revealed high expression of FBXW7 and low expression of c-Myc.

Conclusion: These findings suggest that FBXW7 plays a crucial role in the maintenance of quiescent CSCs resistant to gefitinib in EGFR mutation-positive NSCLC.

Respiratory Infections (non-tuberculosis) 1

A0036

\section{AETIOLOGY OF COMMUNITY ACQUIRED PNEUMONIA WITH FEVER AND THE PRESENTATION AND PROGNOSIS OF VIRAL INFECTION: A PROSPECTIVE OBSERVATIONAL STUDY \\ JIN-FU XU1', RU-JIA TAO ${ }^{1}$, XIAO-LI LUO'1, WEI-JUN CAO}

${ }^{1}$ Department of Respiratory and Critical Care Medicine, Shanghai Pulmonary Hospital, Tongji University School of Medicine, Shanghai, China

Background and Aim: Patients with community acquired pneumonia (CAP) caused by viruses can develop severe complications, which result in hospitalization and death. The purpose of this study was to analyse the aetiology, incidence, clinical characteristics, and outcomes of CAP patients with fever during non-pandemics in one pulmonary hospital.

Methods: A management system was established for monitoring the CAP patients with fever. Multiplex polymerase chain reaction (MPCR) kits were used to detect 10 viruses (influenza $A$ and $B$, adenovirus, respiratory syncytial virus $A$ and $B$, picornavirus, parainfluenza virus, coronavirus, human metapneumovirus, and bocavirus). Data on age, gender, underlying diseases, complications, laboratory indexes, and outcomes were collected by physicians.

Results: This prospective study included 320 patients with fever, $23.4 \%$ were viral-positive by $\mathrm{mPCR}$, with influenza virus most prominent followed by picornavirus. Strong variation in seasonal distribution was shown in viral infections, with peak months from December to February. Patients with influenza infection were likely to be taken to emergency rooms and have respiratory failure with higher creatinine kinase levels and lower white blood cell counts. Streptococcus pneumoniae followed by Haemophilus influenzae were the most common bacteria in viral coinfections, which accounted for one third of virus-positive patients. Coinfection can independently act as a risk factor for the CURB65 severity score, but not for death in hospitalization.

Conclusion: Viruses play an important role in CAP patients with fever, a systematic clinical, radiological and biological analysis of these patients can contribute to effective therapy that may prevent the development of CAP and improve the outcomes. The present work showed the first and an elaborate analysis evidence of viral infection among fever CAP inpatients.

Acknowledgements: The work was supported by the National Natural Science Foundation of China (grant number NSFC 81670006); the Key Project of the Shanghai ShenKang Hospital Development Center (grant number SHDC12014104); and the Shanghai Leading Talent Project (grant number 2016036).

\section{CLINICAL, LABORATORY FEATURES AND OUTCOME OF COMMUNITY-ACQUIRED PNEUMONIA IN ALCOHOLIC PATIENTS IN RESPIRATORY CENTER, BACH MAI HOSPITAL TRANG VU ${ }^{\mathbf{1} 2}$ \\ ${ }^{1}$ Bach Mai Hospital, Hà Nôi, Viet Nam, and ${ }^{2}$ Respiratory Center, Bach Mai hospital, Hanoi, Viet Nam}

Background: Community-acquired pneumonia (CAP) is one of the most common infectious diseases and is an important cause of mortality and morbidity worldwide. Alcoholism has been identified to be a risk factor for CAP, especially severe CAP.

Aims: To describe clinical, laboratory features and outcomes of CAP in alcoholic patients hospitalized in a respiratory center.

Methods: Retrospective descriptive study of CAP hospitalized in Respiratory Center of Bach Mai hospital in Ha Noi, Vietnam, from January 2015 to December 2015. Our study included 419 patients divided into 2 groups: $1^{\text {st }}$ group including 73 alcoholic patients and $2^{\text {nd }}$ group including 346 non-alcoholic patients.

Results: There was a higher rate of male gender in alcoholic group $100 \%$ vs $48.6 \%$ in non-alcoholic group ( $p<0.01)$. The average age of alcoholic patients was $55.3 \pm 9.1$ (years), younger than non-alcoholic patients with $61.0 \pm 19.6$ (years). $31.5 \%$ alcoholic patients suffered from alcohol withdrawal syndromes within 24 hour of admission, with the most common symtomp of tremulousness (95.7\%). The most common symptoms of alcoholic patients were fever, productive cough, dyspnea and crackles, which acounted for $75.3 \%, 71.2 \%, 60.3 \%$ and $75.3 \%$ respectively. Some laboratory data of alcoholic patients was worse than non-alcoholic patients, for detail, higher percentage of anemia, thrombocytopenia, and hypoalbuminemia in alcoholic patients: $80.8 \%$ vs $49.7 \%, 35.6 \%$ vs $14.1 \%, 66.7 \%$ vs $45.0 \%$ respectively $(p<0.05)$. Pathogens were found in only $5.4 \%$ of alcoholic patients, $2 / 3$ of them were Gram negative bacteria. Mortality rate was $30.1 \%$ in alcoholilc group vs $14.7 \%$ in non-alcoholic group. Nearly a half of alcoholic patients had complications (45.2\%) with the most common one of pleural effusion, respiratory failure and abcess.

Conclusions: In our study, alcoholic patients suffered from CAP at the younger age, with worse laboratory test results and higher mortality rate, compared to non-alcoholic patients.

A0038

\section{RISK STRATIFICATION FOR THE DEVELOPMENT OF CHRONIC PULMONARY ASPERGILLOSIS IN PATIENTS WITH MYCOBACTERIUM AVIUM COMPLEX LUNG DISEASE} KOJI FURUUCHI ${ }^{1}$, AKIHIRO ITO ${ }^{1}$, TORU HASHIMOTO ${ }^{1}$, SHOGO KUMAGAI $^{1}$, TADASHI ISHIDA ${ }^{1}$

${ }^{1}$ Department of Respiratory Medicine, Ohara Memorial Kurashiki Healthcare Foundation, Kurashiki Central Hospital, Kurashiki, Japan

Background and Aim: The number of patients with pulmonary nontuberculous mycobacterial disease complicated by chronic pulmonary aspergillosis (CPA) has been increasing recently. Moreover, CPA is reportedly associated with mortality in patients with Mycobacterium avium complex lung disease (MAC-LD). In the present study, we aimed to identify the risk factors for developing CPA and to stratify the risk for CPA development in patients with MAC-LD.

Methods: We retrospectively examined 361 patients who were newly diagnosed with MAC-LD. The risk factors for CPA development were examined using multivariate Cox proportional hazards regression analyses. A risk stratification system was established using the risk factors and receiver operating characteristic curve analyses. 